\title{
Las relaciones sistémicas en la Ciencia del Periodismo
}

\author{
Dra. Hada Miluska Sánchez Gonzales \\ Universidad de Sevilla
}

\begin{abstract}
Resumen
Concebimos la Ciencia desde la interactuación y la organización entre los campos científicos y abordamos un proceso metodológico sistémico ceñido al área de las Ciencias Sociales como respuesta a los diferentes planteamientos realizados a partir de las Ciencias experimentales. Proponemos su aplicación en la Ciencia del Periodismo como método de inducción analítica y de teoría fundada en procesos comunicativos y socioculturales, en relación a sistemas abiertos y heterogéneos, autopoiéticos y autorreferentes que operan en la producción de un sistema con respecto a otro.
\end{abstract}

\section{Abstract}

Science is conceived from the interaction and the organization among the scientific fields. Moreover, as a response to the different analysis realized to start from the experimental sciences, we deal with a systemically and methodological process which is joined to the Social Science. Thus, it is proposed its application into the Journalism Science as a method of analytic induction and a theory based in systems, which are autopoietic and autoreferent, that also operate in the production of a system related to another.

Palabras clave: Ciencia, sistema, teoría, periodismo, elemento, relación, interpenetración, autopoiético, autorreferente, sistémica, sociedad y comunicación.

Key words: Science, system, theory, journalism, element, relate, interpenetration, autopoietic, autoreferent, systemical, society and communication.

I concepto de Ciencia ha sido enmarcado por diversos autores, los cuales han facilitado su definición por medio de la Epistemología. Podemos decir que es considerada como una rama de la filosofía que estudia la investigación científica y su producto, el conocimiento científico. No obstante, autores como Descartes conciben la Ciencia como la sabiduría humana que contribuyen a aumentar el conocimiento de los hombres.

Entenderemos la Ciencia desde la organización relacional entre los campos científicos como un conjunto de reacciones en interactuación. Proceso que produce nuevos resultados de acuerdo a las exigencias de un enfoque transdisciplinario que ha demostrado su fecundidad en la Ciencia.

\footnotetext{
BUNGE, Mario: Epistemología. Ariel, Barcelona, 1985, pág. 13.
} 
De acuerdo con el progreso intelectual que se dio a partir de la crisis de la Segunda Guerra Mundial, surge la Teoría General de los Sistemas de Von Bertalanffy. Propuesta que se ha enfocado al estudio del área de las ciencias experimentales como: Matemática, Física, Biología, etc. Se trata de reducir los campos complejos a través de los procesos dinámicos relacionales, propios de la Sistémica. Tiene como base el "isomorfismo", por cuanto la estructura de un sistema puede ser aplicado para esclarecer otro sistema ${ }^{2}$.

Han sido pocos los autores que han abordado este tema dentro de la Ciencia del Periodismo, cabe citar a los autores-periodistas como: Raúl Rivadeneira, Miguel Urabayen, Javier del Moral y Francisco Esteve Ramírez, quienes desde su parcela de investigación han abordado la Teoría General de los Sistemas de Von Bertalanffy. Estudio que está determinado dentro del área de las Ciencias experimentales, de ahí que pensamos en la necesidad de dar una respuesta sistémica a partir de las Ciencias Sociales. Ello supone, estar a la altura de las demás ciencias con recursos propios de nuestro ámbito de estudio.

En este sentido, validamos la Teoría General de la Sociedad de Niklas Luhmann $^{3}$ como base teórica para proponer la Teoría de los polisistemas de Itamar Even Zohar, que con lleva a la realización de un pensamiento relacional a través de sistemas abiertos, dinámicos y heterogéneos.

Las teorías ${ }^{4}$ se encuentran relacionadas entre sí, por ello constituyen el contenido principal de la Ciencia. Cada campo científico es una parte del pasado, es decir, que toda teoría puede ser sustituida por otra, en razón de que pueda explicar mejor a su objeto de estudio ${ }^{5}$.

De acuerdo a la premisa expuesta, hemos trabajado con la Teoría General de la Sociedad y la de los polisistemas, con el objeto de conceptualizar el notable crecimiento del sector informativo en los medios televisivos, según la supremacía por su audiencia.

Ésta metodología nos ayudará a determinar los elementos y fenómenos adversos dentro de la Ciencia del Periodismo, así como su importancia informativa, significativa y funcional dentro del mensaje. Nos referimos a aquellas unidades que caracterizan la información dentro del contexto actual y, por consiguiente, se encuentran implicadas dentro de la información periodística.

\footnotetext{
2 Ibídem, pág. 41.

3 LUHMANN, Niklas: Sociedad y sistema: la ambición de la teoría. Piadós, Barcelona, 1990.

${ }^{4}$ Señalaremos la conceptualización de teorías como aquellos principios que se elaboran en el trabajo científico, con el objetivo de sistematizar los elementos propuestos, los cuales están formados por expresiones que afirman o niegan al objeto de la Ciencia. SIERRA BRAVO, R: Tesis doctorales y trabajos de investigación científica. Metodología general de su elaboración y documentación. Paraninfo, Madrid, 1996, pág. 45.
}

${ }^{5}$ ALCINA FRANCH, José: Aprender a investigar: métodos de trabajo para la redacción de tesis doctorales. Humanidades y Ciencias Sociales. Compañía Literaria, Madrid, 1994, pág. 22. 


\section{LAS TEORÍAS SISTÉMICAS}

El pensamiento relacional ha llevado a analizar la cultura como un sistema global, como un contiguo heterogéneo donde los seres humanos se organizan.

Planteamos el problema a nivel metateórico, con el fin de explicar la existencia relacional de sí un cierto fenómeno observable pertenece a un conjunto determinado (grupo, campo o sistema), y de si es relevante o puede relacionarse. El análisis sistémico afectará al éxito del "procedimiento frente a otro desde el punto de vista de la suficiencia teórica ${ }^{6}$. Se trata de buscar un tipo de teoría que permita resolver los problemas de la Teoría en vigor.

La evolución histórica de los sistemas y de las teorías relacionales a nivel sistémico, necesitan ser formuladas en orden cósmico. Iniciamos con la frase aristotélica: Él todo es más que la suma de las partes, Objetivo que se enmarca dentro del carácter organizativo de cualquier fenómeno ${ }^{7}$.

Hacia los años treinta, Von Bertalanffy fue quien expuso oralmente el concepto de la Teoría General de los Sistemas, propuesta que tuvo diferentes precursores, como Köhler, quien estuvo a punto de generalizar la Teoría de la Gestalt a la de los sistemas generales. La segunda proposición fue realizada por Lotka, científico que determinó la definición de los sistemas dinámicos a la que otros consideraban como Teoría General. Su estudio se ciñe en los sistemas de ecuaciones diferenciales, adaptadas de forma simultánea, con el fin de constituirse en un nuevo terreno de la ciencia ${ }^{8}$.

Es necesario aplicar el concepto de sistema como grupo para referirnos a todos los ámbitos sociales en el que se encuentra las distintas disciplinas. La Ciencia Social es la ciencia de los sistemas sociales. Por esta razón deberá seguir el enfoque de la ciencia general de los sistemas ${ }^{9}$.

Estas ciencias se encuentran vinculadas al Periodismo, unas más que otras, según el campo de acción específico de cada cual, de ahí que podamos decir que la Sociología entronca con la Psicología y el Periodismo en el estudio social e interactuante con los individuos dentro de un sistema sociocultural donde el sistema sociocultural comunicativo es el protagonista.

6 Even Zohar menciona a Machlup para referirse a la suficiencia teórica en el hecho de elegir la variable adecuada que determine el sistema de funciones. Véase en EVEN ZOHAR, Itamar: "Factores y dependencias en la cultura" en AA.VV.: Teoría de los Polisistemas. Arco, Madrid, 1999.

7 Podemos decir que el "examen de las partes y procesos aislados no puede darnos una explicación completa de los fenómenos vitales". BERTALANFFY, L. V., ROSS ASHBY, W., WEINBERG, G. M.: Tendencias en la Teoría General de Sistemas. Alianza, Madrid, 1987, págs. 29, 33.

8 lb., págs. 35 y 36 .

${ }^{9}$ Nos referimos a: la Sociología, Economía, Ciencia Política, Psicología Social, Antropología Cultural, Lingüística y buena parte de la historia y las humanidades. BERTALANFFY, Ludwing Von: Teoría General de los Sistemas. Fondo de Cultura Económica de México, México, 1986, pág. 204. 
Los sistemas comunicativos nos conducen a determinar conceptos como la retroalimentación informativa y comunicativa, con relación al funcionalismo dinámico.

Nos referimos a los fenómenos sociales situados dentro de un contexto sociocultural caracterizado por su causa y razón que establece algún significado, con el fin de explicar los múltiples aspectos complejamente interconectados en las entidades. Las Ciencias Sociales a través de los sistemas socioculturales forman parte de un universo que ha sido creado por el hombre y que se llama cultura ${ }^{10}$.

El Periodismo, como campo interactuante con otras ciencias, requiere de una metodología funcional propia de los movimientos sistémicos. Estudio reciente en esta disciplina que ha sido vista desde campos experimentales, sin embargo, nosotros la adscribimos a su propio campo de acción a partir de las Ciencias Sociales.

Las ciencias de la información consideran que su objeto y contenido de estudio se incluye dentro de la Ciencia de la Sociología, que es contemplada por las ciencias nomotéticas. Sin embargo, las bases de las ciencias de la información se sustentan en la existencia de las reglas y leyes causales en el campo de los medios $^{11}$.

No obstante, muchos de los que se dedican a su estudio y que pertenecen al campo de los fenómenos de la vida se encuentran perdidos dentro de un gran campo confuso, por el hecho de tener que experimentar y comparar los resultados precisos de investigaciones físicas con las aproximaciones y construcciones parciales de las ciencias sociales ${ }^{12}$, ya que las ciencias de la información son parte de las Ciencias Sociales.

Los fenómenos dentro de un complejo campo de acción pueden ser explicados a través de la Teoría General de los Sistemas en el sentido más estricto. Se intenta partir de una definición general de sistema como complejo de componentes interactuantes, conceptos característicos de totalidades organizadas ${ }^{13}$. Su aplicación se relacionará con los fenómenos específicos.

Se trata de un método integrador, aplicable a los campos del conocimiento científico, en relación con las complejas organizaciones de carácter social o tecnológico ${ }^{14}$.

\footnotetext{
${ }^{10}$ Ibídem, págs. 205 y 206.

${ }^{11}$ Son las ciencias que intentan establecer las leyes ceñidas, algunas veces, a las relaciones cuantitativas, relativas, constantes y expresables. En este sentido, son hechos generales de relaciones ordinales y de análisis estructural. Véase en URABAYEN, Miguel: Estructura de la información. periodística. concepto y método. Mitre, Barcelona, 1988, págs. 59, 60.

${ }^{12}$ CARREL, A.: La incógnita del hombre. Madrid, 1941, pág. 9, en URABAYEN, Miguel: Op. Cit., pág. 103.

${ }^{13}$ BERTALANFFY, Ludwing Von: Op. Cit., pág. 94.

${ }^{14}$ URABAYEN, Miguel: Op. Cit., 1988, pág. 106.
} 
Proceso que es definido como medio ambiente y subsistemas porque el medio es el conjunto de todos los objetos que afectan a los sistemas o que son modificados por la conducta de éste, al ser considerados como parte del medio del otro $^{15}$.

El término de sistema desde su primera conceptualización se ha ido reformando a través del tiempo, con el criterio de establecer relaciones entrelos objetos y entre sus atributos, en el que los objetos son los componentes o partes del sistema. Los atributos son las propiedades de los objetos y las relaciones mantienen unido al sistema. Las formas y las jerarquías sistémicas ayudarán a plantear el nivel de entitividad enfatizando en los grados de su organización.

Reconocemos la aparición de recientes modelos conceptuales en que se divide el pensamiento y por lo tanto la investigación, en tanto surjan nuevos fenómenos sociales que impliquen su reconocimiento científico.

Con la Teoría General de los Sistemas aparecen nuevos enfoques como el movimiento cibernético, en relación con los proceso de retroalimentación hacia los sistemas dinámicos. Se produce la interacción entre comunidades con intereses similares de tipo organizativo y conductual. Su elaboración se debe a los problemas existentes en los propios sistemas y en la tecnología del control de la comunicación como construcciones representativas a nivel cognitivo ${ }^{16}$.

El sistema funcional hace patente la presencia del tiempo en la teoría dinámica y de control, y por otro, el proceso de las traducciones. Concepción que ha ido surgiendo desde su iniciación como la formulación formal de un lenguaje hacia otro ${ }^{7}$.

No cabe duda que las investigaciones han ido abriendo nuevos caminos en los sistemas de interacción, si bien las relaciones con los hechos empíricos siguen siendo tenues, la Teoría de la Información ha sido un buen aporte para el campo tecnológico ${ }^{18}$.

La tecnología de la información nos permite comprender la estructura de los medios, a partir de la transformación tecnológica de la información periodística con Gutemberg $^{19}$ y la imprenta, que hasta la fecha no ha dejado de perfeccionarse ${ }^{20}$.

Según las premisas antes expuestas, pretendemos esclarecer el concepto de sistema como un proceso metodológico interaccional dentro de la Ciencia del Periodismo.

${ }^{15}$ WATZLAWICK, Paúl, BEAVIN BAVELAS, Janett, y JACKSON, Don: Teoría de la comunicación humana: interacciones, patologías y paradojas. Herder. Barcelona, 1989, pág.118.

${ }^{16}$ BertalanfFy, L. V., RosS AShBY, W., WeINBERG. GM: Op. Cit., págs. 37 y 40.

17 Ibídem, págs. 43 y 44.

18 BERTALANFFY, Ludwing Von: Op. Cit., pág. 103.

${ }^{19}$ Véase en GÓMEZ Y MÉNDEZ, José Manuel: Gutemberg.es. Colectivo Gallo de Vidrio, ㄲo 4, Sevilla, 2000.

${ }^{20}$ URABAYEN, Miguel: Op. Cit., pág. 77. 


\section{LA SISTÉMICA EN LA CIENCIA DEL PERIODISMO}

Los diversos cambios y evoluciones en las ciencias, nos llevan a aplicar las relaciones sistémicas como propuesta metodológica dentro de la Ciencia del Periodismo. No obstante, mencionaremos algunos autores como Raúl Rivadeneria, Miguel Urabayen, Javier Fernández del Moral y Francisco Esteve Ramírez, quienes desde sus parcelas de investigación han abordado la Teoría General de los Sistemas.

Raúl Rivadeneira, en su libro titulado: Periodismo: la Teoría General de los Sistemas y la Ciencia de la Comunicación, dedica un apartado al periodismo impreso como sistema. Cita a Charles Peirce para referirse a las señales comunicacionales en la concepción pragmática de su funcionalidad establecida en las relaciones sistémicas. Más tarde, autores como Morris y Carnap amplían estas conceptualizaciones profundizando en la Teoría de los Sistemas. Se trata de autores que son ejemplo de la diversificación científica para el análisis de los instrumentos de la comunicación humana, porque el estudio del periodismo es inseparable del de la comunicación humana, lo es menos todavía del sistema de la comunicación de masas ${ }^{21}$. El Periodismo pertenece al proceso sistémico donde se da la interacción comunicativa y por lo tanto, transdisiciplinar.

La aplicación sistémica de Rivadeneira nos introduce a la Teoría General de los Sistemas propuesta por Bertalanffy, con relación a los procesos de naturaleza biológica, psicológica y sociológica. Adapta la propiedad del sistema abierto con relación a sus jerarquías y logra distinguir dos tipos de relación. La primera se efectuará entre los elementos componentes, y la segunda, entre otros sistemas del entorno.

Un sistema se establece por elementos constituidos dentro de un ambiente y entorno, y a su vez, sin exclusivismo alguno debido a la presencia de sistemas interactuantes y organizados. No es pensable un objeto sin su entorno y dentro de éste, sin las nociones de función y relación ${ }^{22}$.

Rivadeneira reconoce que los sistemas se constituyen en relación con las categorías, de ahí que denomina a un primer grupo como superiores porque abrirán más de un sistema, el segundo serán los concretos, el tercero llamado subsistema. Y el cuarto y último grupo está formado por cada uno de los grados en que se dividen los subsistemas ${ }^{23}$.

Por su parte, Miguel Urabayen, reconoce la Teoría General de los Sistemas dentro de los medios de información por medio de modelos matemáticos. Define la disciplina de la estructura de la información periodística y reconoce la relación entre estructura y sistema ${ }^{24}$.

${ }^{21}$ RIVADENEIRA PRADA, Raúl: El periodismo: la Teoría general de los sistemas y la ciencia de la comunicación. Trillas, México, 1977, págs. 26 y 27.

${ }^{22}$ WATZlAWICK, Paúl, BEAVIN BAVElAS, Janett, y JACKSON, Don: Op. Cit., pág. 33.

${ }^{23}$ RIVADENEIRA PRADA, Raúl: Op. Cit., pág. 34.

${ }^{24}$ En su libro titulado: Estructura de la información periodística: concepto y método antes citado. 
Javier Fernández del Moral también hace centra en la Teoría de Bertalanffy. No obstante, señala la importancia de los medios de comunicación como integrante de un gran sistema donde se ubica la Ciencia del Periodismo. Coincide con los planteamientos de autores como Rivadeneira y Urabayen antes mencionados.

Relaciona la ciencia, la sociedad y el hombre, con referencia a la influencia ejercida por los modelos comunicativos. Se centra en la posibilidad de reducir el proceso integrado de las diferentes áreas de la especialidad científica con respecto a la sociedad. Utiliza la Teoría General de la Información, la informática y el proceso de comunicación, ciencias que se apoyan en otras ciencias ${ }^{25}$.

El modelo se distingue en función de tres pasos particulares y con un criterio integrador: del especialista a la ciencia, luego de cada ciencia concreta al conjunto de todas las ciencias experimentales, y de éstas a la sociedad. No obstante, insisten en la necesidad de introducir una propuesta correcta de comunicación entre los diferentes pasos. ${ }^{26}$.

Posteriormente Javier Fernández del Moral conjuntamente con Francisco Esteve Ramírez tratan la Teoría General de los Sistemas partiendo de la necesidad de la especialización y de la comprobación de relaciones isomorficas y analogías de conceptos y métodos, que son resueltos en el modelo de un sistema. Construyen la estructura sistémica de la información periodística especializada enfocada a la pluralidad informativa ${ }^{27}$.

Las propuestas de los autores como Rivadeneira, Urabayen, Fernández del Moral y Esteve corresponden a los primeros movimientos sistémicos periodísticos fundamentados a través de la Teoría General de los sistemas de Von Bertalanffy. Su tendencia nos ayudará a abordar una Teoría centrada en los campos científicos que nos lleve acercarnos a la unidad de la Ciencia y a su integración.

En consecuencia, la Teoría General de los Sistemas es aplicada a los diferentes campos científicos porque permiten establecer las relaciones isomórficas entre las distintas ciencias, por el hecho de intercambiar experiencias.

\footnotetext{
${ }^{25}$ FERNÁNDEZ DEL MORAL, Javier: Modelos de comunicación científica para una información periodística especializada. Dossat, Barcelona, 1983, pág. 106.

${ }^{26}$ Ibídem, pág. 162.

${ }^{27}$ Véase el libro de los autores citados: Fundamentos de la información periodística especializada, Síntesis, Madrid, 1996. Distinguen diferentes teorías isomórficas para cimentar sus bases teóricas y modelos conceptuales sobre la Teoría General de los Sistemas de Bertalanffy.
} 


\section{PROPUESTA SISTÉMICA A PARTIR DE LAS CIENCIAS SOCIALES Y EN EL PERIODISMO EN PARTICULAR}

Es necesario aplicar una teoría propia de nuestro ámbito de estudio. Preocupación ${ }^{28}$ reflejada por muchos autores ante la necesidad de establecer una relación entre la Teoría General de los Sistemas y las Ciencias Sociales para una correcta comprensión y utilización de las mismas.

Problemática que nos lleva a proponer teorías sistémicas generadas a partir de las Ciencias Sociales como respuesta a las necesidades funcionales de la Ciencia del Periodismo. Reducimos de esta manera las complejidades de estudios posteriores sobre fenómenos informativos y sus mensajes.

La presencia de un funcionalismo dinámico nos lleva a citar la Teoría General de la Sociedad de Niklas Luhmann. Asumimos las exigencias transdiciplinares que queda marcada por dos elementos: lo novedoso y el deseo de obtener ganancias por la fuerza que se explicita de la propia teoría. Debe ser tratada como un arma que tiene como fin reducir dicha complejidad ${ }^{29}$.

La observación dentro de un entorno es fundamental porque se encuentra unido los sistemas autorreferentes sobre sí mismo dentro de un tiempo que permite su clausura. Se diferencian respecto a su entorno por sus leyes de observación y por la construcción de su modo de actuar ${ }^{30}$.

La sociedad está compuesta por comunicaciones, que son sistemas autorreferentes. Éstos tienen en la conciencia y en el lenguaje su propio modo de operación autopoiética, con relación al entorno cultural del individuo ${ }^{31}$.

La sociedad se diferencia según su grado de evaluación y desarrollo en diferentes subsistemas sociales, que son sistemas autorreferentes y autopoiéticos ${ }^{32}$. Se especializan en el ámbito de sus comunicaciones, y en el hecho de resolver las complejidades del sistema, observándose a sí mismos y a otros sistemas. Se trata de disolver las esencias estáticas considerando la relación y la diferencia por medio de la observación. Este proceso fronterizo resguarda los límites de un sistema y determina su creación de forma específica.

${ }^{28}$ Fernández del Moral y Esteve argumentan tal necesidad en su libro titulado: Fundamentos de la información... antes citado.

29 LUHMANN, Niklas: Op. Cit., págs. 11, 14 y 16.

30 Ibídem, págs. 21, 24.

${ }^{31}$ Luhmann tiene en cuenta: "El concepto de sujeto antropológico en un sistema autorreferente basado en la conciencia y en el lenguaje. Plantea una nueva perspectiva de análisis sobre un conjunto de conceptos tradicionalmente dependientes del concepto clásico de sujeto". Ib., pág. 27.

${ }^{32}$ Vamos a decirlo de forma clara: "la autorreferencialidad alude directamente a la formación de las propias estructuras en el sistema; la autopoiesis hace referencia a todo lo que acontecen el sistema como operación (lo cual incluye también las estructuras)". LUHMANN, Niklas: Sistemas sociales: lineamientos para una teoría general. Anthropos, Barcelona, 1998. 
Dentro del sistema y del pensamiento híbrido de la época actual y en conexión relacional con los sistemas dinámicos; el siguiente paso sistémico nos han lleva a analizar la cultura como un sistema global, de ahí que planteamos la Teoría de los Polisistemas de Even Zohar como una teoría acorde con la problemática de hoy. Proceso que nos ayudará a establecer una red relacional polisistémica, acorde a las necesidades de investigaciones emergentes.

Esta Teoría se presenta como una continuación del funcionalismo dinámico ${ }^{33}$. La propuesta se establece como un vínculo entre la heterogeneidad del sistema, el cambio por una parte y la estructura por otra. La Teoría de los Polisistemas revela el pensamiento relacional y el concepto de un sistema abierto, dinámico y heterogéneo ${ }^{34}$.

Esta teoría nos ayudará a esclarecer el proceso sistémico en el medio televisivo, donde las diversas ciencias realizan una diferenciación sistémica. Interacción que se determina examinando su entorno y su unidad de acción con relación a los procesos sociales.

Establecemos los límites entre las ciencias siempre y cuando se encuentren definidas como elementos que forman parte del sistema o de su entorno, con el criterio de separar los elementos, más no su relación ${ }^{35}$.

Estimamos la diferenciación sistémica de acuerdo a los límites definidos entre las ciencias y los consideramos como elementos determinados y, a su vez, abiertos de demarcación de límites o de atribución de acontecimientos hacia dentro y hacia fuera. Son tratados como medios propios del sistema que no sólo se adaptan a su entorno, sino a su propia complejidad a través de los procesos de "diferenciación interna del sistema".

La Teoría de los Polisistemas en su tesis de constitución múltiple, permite que se profundice el concepto actual de la comunicación que se halla señalado por el enmarañado proceso que sigue el orden de la sociedad.

${ }^{33}$ Even Zohar se refiere al "formalismo ruso del estructuralismo checo, de la semiótica soviética, etc.". Véase en EVEN ZOHAR, Itamar: La función de la literatura en la creación de las naciones de Europa, traducción de M. Iglesias Santos, en Villanueva, D.: Avances en teoría de la literatura. Servicio de publicaciones de la Universidad de Santiago de Compostela, Santiago de Compostela, 1990, págs. 357, 377. Posteriormente apareció una versión inglesa.

${ }^{34}$ Podríamos afirmar que, a partir de los años veinte, la teoría ha hecho casi inevitable sus comienzos con el desarrollo de herramientas "conceptuales para un amplio complejo de fenómenos". Cita una serie de ejemplos, entre ellos las obras de Bogatyrev y Mukarovsky en la semiótica cultural, así mismo las últimas contribuciones de la Escuela de Tartu - Moscú. Ibídem, pág. 26.

${ }^{35}$ LUHMANN, Niklas: Op. Cit., pág. 79.

${ }^{36}$ Para mayor referencia, véase en BUCKLEY, Walter: Modern Systems Research for the Behavioural Scientist. Chicago, 1968, pág. 108-118, en LUHMANN, Niklas: Op. Cit., pág. 101. 


\section{APLICACIÓN SISTÉMICA}

El concepto de Comunicación exige selecciones por motivos de complejidad porque se establecen dentro de un sistema donde se complica la temática entre sistema y entorno. Su relación con respecto a las demás ciencias exige un planteamiento sistémico que permita la ejecución propia de la evolución de los sistemas sociales articulada por sus procesos ${ }^{37}$.

Establecemos el método a través de un análisis funcional sujeto a la información, donde existen afinidades con determinadas predisposiciones conceptuales de la teoría que apunta hacia los intereses del conocimiento que se anuncian con conceptos de complejidad, contingencia y selección. El objetivo de esta relación comprende lo existente como contingente y lo distinto como comparable ${ }^{38}$.

La ordenación sistémica se inicia en nuestro ámbito de estudio, es decir, desde las Ciencias Sociales ${ }^{39}$, con el fin de profundizar, afianzar y crecer como Ciencia.

Abordamos las conceptualizaciones de Niklas Luhmann sobre las relaciones entre los sistemas y su entorno para acoger la Teoría de los Polisistemas de Even Zohar a partir de una relación sistémica, abierta, dinámica y heterogénea.

La intervención del sistema y del entorno sustituye la diferencia entre todo y parte por la de identidad y diferencia como la constante constitución de subsistemas en el interior de sistemas, porque sin conciencia de sistemas psíquicos apenas se da la comunicación ${ }^{40}$.

Es necesario hacer una distinción sistémica entre sistema y entorno, y elemento y relación. En ambos casos se trata de la unidad de la diferencia como constitutiva, es decir, de la misma manera que no existe sistemas, sin entorno, tampoco existen elementos sin conexión relacional, ni relaciones sin elementos ${ }^{41}$.

Se trata de reducir la complejidad del sistema periodístico de un modo especializado porque la sociedad se diferencia en diferentes subsistemas. Nos referimos a la Lingüística, la Retórica, la Sociología, la Educación, etc.

La relación sistémica nos lleva a determinar dentro del medio televisivo, a la Ciencia del Periodismo y a su interactuación comunicativa y de orden social, con respecto a las demás. Se trata de relaciones funcionales, de sistemas abiertos dinámicos y heterogéneos concebidos a través de la Teoría de los Polisistemas de Even Zohar.

\footnotetext{
37 LUHMANN, Niklas: Op. Cit., pág. 102.

${ }^{38}$ Ibídem, pág. 131.

${ }^{39}$ Nos referimos a la "Teoría General de la Sociedad", significa partir de una teoría propia de nuestro ámbito de estudio.

${ }^{40}$ LUHMANN, Niklas: Op. Cit., pág. 54.

${ }^{41}$ Ibídem, pág. 59.
} 
Abordamos el caso específico del Periodismo y la Psicología en relación a elementos comunes a sí mismos que se construyen a partir de un fundamento existente y justo con relación al otro. Fórmula sistémica que se produce teniendo en cuenta los sistemas abiertos y autopoiéticos porque operan en la producción de un sistema con respecto al otro.

La dificultad sistémica se reducirá mediante el establecimiento de sus límites, siempre y cuando se encuentren definidas como elementos que forman parte del sistema o de su entorno ${ }^{42}$.

Se trata de dos Ciencias que logran ramificar su estudio, reconociendo la integración de unos elementos con respecto a otros. Nos referimos a los elementos de la psicología fenomenológica inmersos en el campo de la Psicología, los cuales serán interpenetrados en el sistema del Periodismo dentro del medio televisivo ${ }^{43}$.

La información periodística condicionan el lenguaje de los sentimientos dentro de sus componentes: visual, sonoro y verbal. En tanto, en la Ciencia de la Psicología, la Psicología fenomenológica es la vertiente que se aproxima al estudio de la emotividad ${ }^{44}$.

La diferenciación nos permite reducir la diversidad a la que se enfrenta el Periodismo respetando la independencia de cada uno de ellos. Relación que se conoce con el nombre de interpenetración.

Hablar de interpenetración significa concebir como opera un sistema. Proceso que pone a disposición de otro su propia estructura para que pueda seguir construyéndose la complejidad que le es propia ${ }^{45}$.

Los movimientos sistémicos nos permiten interpenetrar a la emotividad dentro del sistema periodístico. Su importancia se centra en la acción y reacción dentro del sistema en cuanto a las relaciones que se establezcan, y a través de un solo uso sistémico.

42 lb., pág. 79.

${ }^{43}$ El vínculo relacional entre el Periodismo y la Psicología se inicia en "la observación del hecho fenomenológico, a partir de los procesos cíclicos entre la información y la percepción a nivel cognitivo, posteriormente a través de su técnica de uso por medio de la conciencia humana, con relación al sentido técnico de progreso de ambas ciencias en tiempo y espacio". SÁNCHEZ GONZALES: H. Miluska: "La relación funcional sistémica entre dos ciencias en el medio televisivo: Periodismo y Psicología” en GÓMEZ Y MÉNDEZ, José Manuel (ed.): Entre la Formación y la Comunicación. Est Libri, Huelva, 2005, págs. 309-333.

${ }^{44}$ Ibídem, págs. 309-333.

${ }^{45}$ LUHMANN, Niklas: Op. Cit., pág. 25. 
Aclaramos la acción del proceso por medio de la Teoría de la acción ${ }^{46}$, porque las acciones deben su unidad a la red de relaciones del sistema que se constituye. Su consecuencia más importante es la producción de una nueva interdependencia entre la disolución y la reproducción de elementos ${ }^{47}$.

Los procesos sistémicos han reducido la complejidad interactuante y de orden comunicativo y social de los sistemas dentro del medio televisivo respetando sus límites y a su vez, facilitado la abstracción de elementos de un sistema hacia otro.

La Ciencia del Periodismo dentro de las relaciones funcionales con la Psicología abstrae al elemento emotivo dentro de la información. El tiempo facilitará la relación de los sistemas, así como el sentido de los cambios que se protagonicen. Hecho que ha propiciado la agregación de un nuevo elemento dentro de su unidad como sistema.

Proceso sistémico que nos lleva a reconocer a un activismo cultural denominado como Periodismo emotivo, el cual da inicio a una nueva etapa dentro de dicha Ciencia haciendo uso de un lenguaje y paradigma códico, según las necesidades de su audiencia. Visualicemos el esquema anexo.

La relación sistémica en la Ciencia del Periodismo y la interpenetración de elementos emotivos.

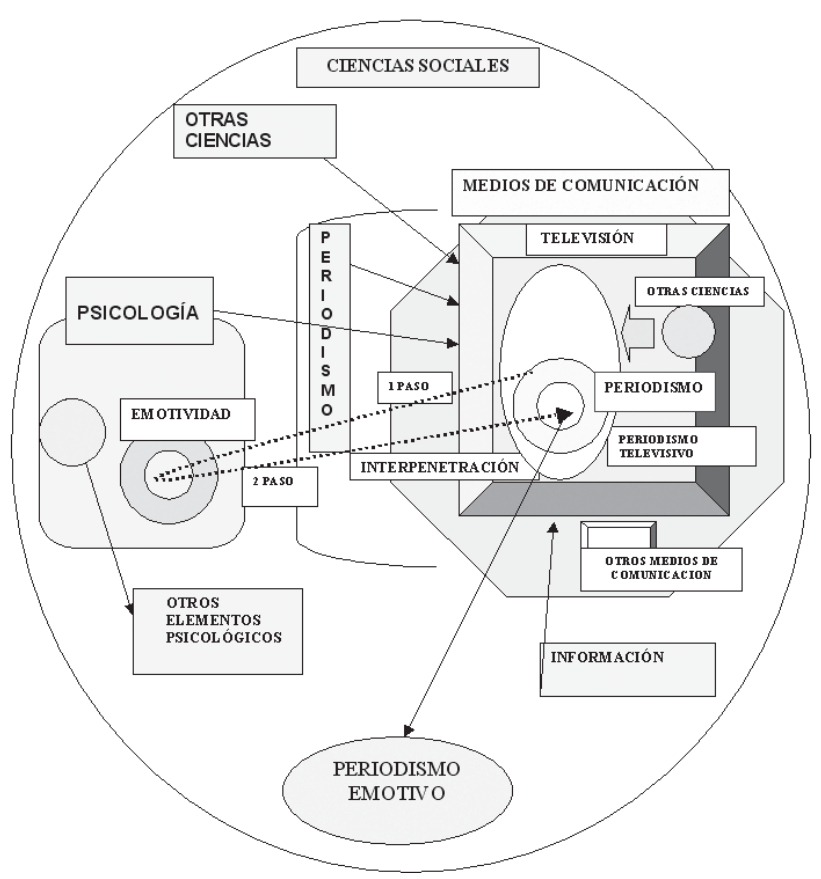

${ }^{46}$ Véase en BUCETA FACORRO, Luis: Fundamentos psicosociales de la información. Centros de Estudios Ramón Areces, Madrid, 1992.

47 LUHMANN, Niklas: Op. Cit., págs. 62, 108, 122. 


\section{BIBLIOGRAFÍA:}

ALCINA FRANCH, José: Aprender a investigar: métodos de trabajo para la redacción de tesis doctorales. Humanidades y Ciencias Sociales. Compañía Literaria, Madrid, 1994.

BERTALANFFY, L. V., ROSS ASHBY, W., WEINBERG, G. M.: Tendencias en la Teoría General de Sistemas. Alianza, Madrid, 1987.

BERTALANFFY, Ludwing Von: Teoría General de los Sistemas. Fondo de Cultura Económica de México, México, 1986.

BUCETA FACORRO, Luis: Fundamentos psicosociales de la información. Centros de Estudios Ramón Areces, Madrid, 1992.

BUNGE, Mario: Epistemología. Ariel, Barcelona, 1985, pág 13.

EVEN ZOHAR, Itamar: "Factores y dependencias en la cultura" en AA.VV.: Teoría de los Polisistemas. Arco, Madrid, 1999.

FERNÁNDEZ DEL MORAL, Javier: Modelos de comunicación científica para una información periodística especializada. Dossat, Barcelona, 1983.

FERNÁNDEZ DEL MORAL, Javier y ESTEVE RAMíREZ, Francisco: Fundamentos de la información periodística especializada, Síntesis, Madrid, 1996.

GÓMEZ Y MÉNDEZ, José Manuel: Gutemberg.es. Colectivo Gallo de Vidrio, 끄 4, Sevilla, 2000.

\section{LUHMANN, Niklas:}

-Sociedad y sistema: la ambición de la teoría. Piadós, Barcelona, 1990.

-Sistemas sociales: lineamientos para una teoría general. Anthropos, Barcelona, 1998.

RIVADENEIRA PRADA, Raúl: El periodismo: la teoría general de los sistemas y la ciencia de la comunicación. Trillas, México, 1977.

SÁNCHEZ GONZALES: H. Miluska: "La relación funcional sistémica entre dos ciencias en el medio televisivo: Periodismo y Psicología” en GÓMEZ Y MÉNDEZ, José Manuel (ed.): Entre la Formación y la Comunicación. Est Libri, Huelva, 2005.

SIERRA BRAVO, R: Tesis doctorales y trabajos de investigación científica. Metodología general de su elaboración y documentación. Paraninfo, Madrid, 1996.

URABAYEN, Miguel: Estructura de la información periodística: concepto y método. Mitre, Barcelona, 1988.

WATZLAWICK, Paúl, BEAVIN BAVELAS, Janett, y JACKSON, Don: Teoría de la comunicación humana: interacciones, patologías y paradojas. Herder, Barcelona, 1989.

(Recibido el 3-02-06, aceptado el 25-07-06) 